\title{
Microwave testing of high-Tc based direct current to a single flux quantum converter
}

Kaplunenko, V. K.; Fischer, Gerd Michael; Ivanov, Z. G.; Pedersen, Niels Falsig; Claeson, T.; Mygind, Jesper; Wikborg, E.

Published in:

Journal of Applied Physics

Link to article, DOI:

$10.1063 / 1.358383$

Publication date:

1994

Document Version

Publisher's PDF, also known as Version of record

Link back to DTU Orbit

Citation (APA):

Kaplunenko, V. K., Fischer, G. M., Ivanov, Z. G., Pedersen, N. F., Claeson, T., Mygind, J., \& Wikborg, E. (1994). Microwave testing of high-Tc based direct current to a single flux quantum converter. Journal of Applied Physics, 76(10), 5996-6000. https://doi.org/10.1063/1.358383

\section{General rights}

Copyright and moral rights for the publications made accessible in the public portal are retained by the authors and/or other copyright owners and it is a condition of accessing publications that users recognise and abide by the legal requirements associated with these rights.

- Users may download and print one copy of any publication from the public portal for the purpose of private study or research.

- You may not further distribute the material or use it for any profit-making activity or commercial gain

- You may freely distribute the URL identifying the publication in the public portal 


\title{
Microwave testing of high- $T_{c}$ based direct current to a single flux quantum converter
}

\author{
V. K. Kaplunenko a) \\ Physics Department, Technical University of Denmark, DK-2800 Lyngby, Denmark and Department \\ of Physics, Chalmers University of Technology, S-41296 Gothenburg, Sweden \\ G. M. Fischer ${ }^{\text {b) }}$ \\ Physics Department, Technical University of Denmark, DK-2800 Lyngby, Denmark \\ Z. G. Ivanov \\ Department of Physics, Chalmers University of Technology, S-41296 Gothenburg, Sweden \\ N. F. Pedersen \\ Physics Department, Technical University of Denmark, DK-2800 Lyngby, Denmark \\ T. Claeson \\ Department of Physics, Chalmers University of Technology, S-41296 Gothenburg, Sweden \\ J. Mygind \\ Physics Department, Technical University of Denmark, DK-2800 Lyngby, Denmark \\ E. Wikborg \\ Ericsson Telecom, S-12625 Stockholm, Sweden
}

(Received 10 January 1994; accepted for publication 29 July 1994)

\begin{abstract}
Design, simulation, and experimental investigations of a direct current to a single flux quantum converter loaded with a Josephson transmission line and driven by an external $70 \mathrm{GHz}$ microwave oscillator are reported. The test circuit includes nine $\mathrm{YBaCuO}$ Josephson junctions aligned on the grain boundary of a $0^{\circ}-32^{\circ}$ asymmetric $\mathrm{Y}^{-} \mathrm{ZrO}_{2}$ bicrystal substrate. The performance of such converters is important for the development of the fast Josephson samplers required for testing of high $-T_{c}$ rapid single flux quantum circuits in high-speed digital superconducting electronics.
\end{abstract}

\section{INTRODUCTION}

Significant progress has been made in the development of rapid single flux quantum (RSFQ) circuits based on low- $T_{c}$ superconductors. ${ }^{1-8}$ Recently the rescarch has bcen extended to high- $T_{c}$ superconducting materials. ${ }^{9,10}$ The realization of stable clocking is one of the basic obstacles in RSFQ circuits. The straightforward candidate, the on-chip integrated free-running high- $T_{c}$ junction Josephson oscillator, cannot provide a sufficiently narrow linewidth of the emitted radiation due to its rather large dynamic resistance. An alternative approach is to use an external narrow-band oscillator such as, e.g., the flux flow oscillator, ${ }^{11}$ 1-dimensional $^{12}$ or 2 -dimensional ${ }^{13}$ arrays of mutually locked Josephson junctions, or even a semiconductor based cryogenic clock oscillator. The electromagnetic coupling of any of these generators to the RSFQ circuit imposes practical as well as basic constraints on the clocking of RSFQ devices. A possible solution to the clocking problem may be to sharpen the input pulses down to, say, $10 \mathrm{ps}$ with the aid of a shock wave propagating on a transmission line based on high- $T_{c}$ Josephson junctions. ${ }^{9}$

It is of general physical interest to test the applicability of the classical low- $T_{c}$ direct current (dc) to the single flux quantum (dc/SFO) converter concept ${ }^{1-3}$ in circuits made of

\footnotetext{
a) Permanent address: Institute of Radio Engineering and Electronics, Russian Academy of Sciences, Moscow, Russia.

${ }^{b}$ Permanent address: Lehrstuhl Experimentalphysik II, Physikalisches Institut, Universität Tübingen, D-72076 Tübingen, Germany.
}

high- $T_{c}$ materials. The major differences are that the high- $T_{c}$ superconductors potentially allow for a much higher operational frequency, and that the circuit design is subjected to severe limitations due to the rather premature level of the multilayer technology with the existing high- $T_{c}$ superconducting materials.

Finally, using the Josephson junction based sampler ${ }^{14}$ a fast dc/SFQ converter will be an important experimental tool for determination of the ultimate performance of RSFQ digital devices. ${ }^{15}$

\section{EXPERIMENTAL SYSTEM AND FABRICATION TECHNIQUE}

The layout of the experimental chip is shown in Fig. 1. It consists of nine $\mathrm{YBaCuO}$ Josephson junctions: the $\mathrm{dc} / \mathrm{SFQ}$ converter $\left(\mathrm{J}_{1}, \mathrm{~J}_{2}\right.$ in Fig. 2$)$ and the RSFQ transmission line $\left(J_{3}-J_{9}\right)$. The nine junctions that are aligned along the grain boundary ${ }^{16}$ in a $0^{\circ}-32^{\circ}$ asymmetric $\mathrm{Y}^{-\mathrm{ZrO}_{2}}$ bicrystal substrate are dc current biased in parallel.

The laser deposited YBCO base electrode $(0.24 \mu \mathrm{m})$ is patterned by photolithography and argon ion milling. The width of the smallest Josephson junction is $5 \mu \mathrm{m}$ and the minimum length of the junctions across the grain boundary is $10 \mu \mathrm{m}$. The dc/SFQ converter and the RSFQ transmission line are (apart from the inductance $L_{1}$ ) covered by a superconducting $\mathrm{Pb} / \mathrm{Au} / \mathrm{Cr}$ ground plane $(0.32 \mu \mathrm{m})$ placed on an insulating, amorphous $\mathrm{SrTiO}_{3}$ layer $(0.5 \mu \mathrm{m})$. These layers are patterned by lift-off processes. 

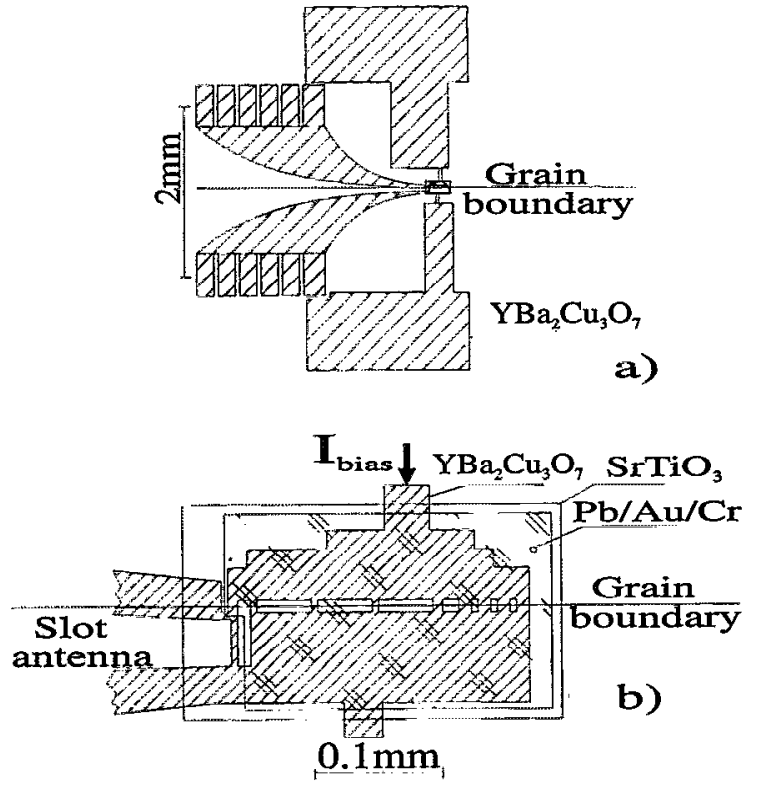

FIG. 1. (a) The circuit shows the dc/SFQ converter and the Josephson transmission line in the center. To the left is the slot antenna matching the 70 $\mathrm{GHz}$ waveguide if signal to the converter; top and bottom are the contact pads used for four-point dc measurements. The enlarged central part (b) shows the nine parallel dc-biased $\mathrm{YBaCuO}$ Josephson junctions $\left(\mathrm{J}_{1}-\mathrm{J}_{9}\right.$ from left to right) straddling the grain boundary. $\mathrm{A} \mathrm{Pb} / \mathrm{Au} / \mathrm{Cr}$ superconducting groundplane, isolated from the $\mathrm{YBCO}$ layer by an $\mathrm{SrTiO}_{3}$ layer, is implemented to reduce the inductances.

The rf signal is transmitted from a room-temperature microwave setup via a standard rectangular waveguide (WR12: $60-90 \mathrm{GHz}$ ) that is matched to the dc/SFQ converter by means of the on-chip integrated coplanar fin-line antenna also shown in Fig. 1. The chip is inserted into two 0.6-mmwide slots that are cut symmetrically in the middle of the broad walls of the waveguide so that the plane of the antenna is parallel to the direction of the $E$ field. The slots extend 8 $\mathrm{mm}$ back from the open end of the waveguide and the position of the chip and hence the antenna can be adjusted in

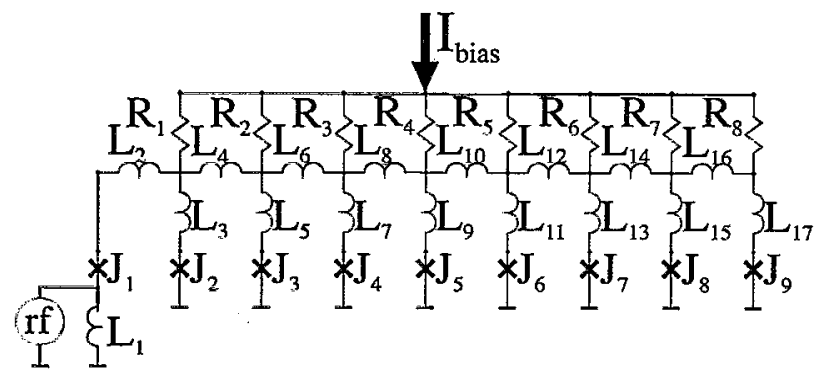

FIG. 2. Equivalent circuit of dc/SFQ converter $\left(\mathrm{J}_{1}-\mathrm{J}_{2}\right)$ and RSFQ transmission line $\left(J_{3}-J_{9}\right)$. The if signal is coupled inductively through $L_{1}$ to the $\mathrm{dc} / \mathrm{SFQ}$ converter (loops $L_{1} \mathrm{~J}_{1} L_{2} L_{3} \mathrm{~J}_{2}$ ). Note that $\left(\mathrm{J}_{1}-\mathrm{J}_{2}\right)$ are dc biased in parallel while if is biased in series. The resistances $R_{1,2,3}=20 \Omega, R_{4}=13.3$ $\Omega, R_{5,6,7,8}=10 \Omega$ were used (only) in the simulation to provide a uniform biasing. All other parameters were chosen close to their experimental values at $4.2 \mathrm{~K}$ and were: critical currents $I_{2,3,4}=52 \mu \mathrm{A}, I_{1,5}=79 \mu \mathrm{A}, I_{6,7,8,9}=105$ $\mu \mathrm{A}$; inductances $L_{1}=21 p \mathrm{H}, L_{2}=16 p \mathrm{H}, L_{3,5,7}=5.2 p \mathrm{H}, L_{4,6}=5.7 p \mathrm{H}$, $L_{8}=6.0 p \mathrm{H}, L_{9}=3.6 p \mathrm{H}, L_{11,13,15,17}-2.3 p \mathrm{H}, L_{10}=3.1 p \mathrm{H}, L_{12,14,16}=2.3$ $p \mathrm{H}$. order to optimize the coupling. The frequency of the applied millimeter wave signal can be tuned in the range 66-74 $\mathrm{GHz}$. The spectral linewidth is less than $100 \mathrm{kHz}$ and the power level can be varied with a precision rotary-vane attenuator.

The sample is mounted on a temperature controlled thermoblock installed inside a cryoprobe which in turn is immersed in a standard liquid helium glass cryostat enclosed in two cylindrical Permalloy cans and a copper shield. The temperature can be regulated in the range $2<T<100 \mathrm{~K}$. All leads connecting to the sample are filtered twisted pairs and the whole setup is carefully electrically shielded. Battery powered supplies were used for all dc bias currents. The measurements were made in a HF shiclded room.

\section{EQUIVALENT CIRCUIT AND SIMULATIONS}

Figure 2 shows the equivalent circuit used in the numerical simulations. The parameters of the elements involved were chosen close to their experimental values at $T=4 \mathrm{~K}$, except for the resistances $R_{1}-R_{8}$ which were included to provide a uniform bias current distribution.

Similar to the classical dc/SFQ converter ${ }^{2}$ the high- $T_{c}$ element consists of two Josephson junctions $J_{1}$ and $J_{2}$ and three inductances $L_{1-3}$. The Josephson junctions are dc biased in parallel and if connected in series. This asymmetric two-junction interferometer has two stable superconducting stales. Biased below the critical current of the smaller junction $\mathrm{J}_{2}$, an increasing if current, $I_{\mathrm{rf}}$, through the inductance $L_{1}$ induces a magnetic field in the loop holes $L_{1} \mathrm{~J}_{1} L_{2} L_{3} \mathrm{~J}_{2}$. Thus the superconducting interferometer produces a clockwise shielding current, which in junction $\mathrm{J}_{2}$ is directed with and in junction $J_{1}$ against the dc bias current. When the if current reaches a threshold level junction $\mathrm{J}_{2}$ switches to the normal state. A fluxon is released to the Josephson transmission line $J_{3-9}$, and an antifluxon with a counter-clockwise circulating current is produced in the loops $L_{1} \mathrm{~J}_{1} L_{2} L_{3} \mathrm{~J}_{2}$, reducing the current in $J_{2}$ below its critical value. When the rf current decreases the thereby induced countcr-clockwise shielding current switches junction $J_{1}$, that releases the associated antifluxon off the loop and resets the interferometer.

The practical layout of the high- $T_{c}$ circuit is significantly different from the low- $T_{c} \mathrm{dc} / \mathrm{SFQ}$ converter layout. Contrary to the $\mathrm{Nb}$-based circuits the odd-numbered inductances $\left(L_{3-17}\right)$ in series with the junctions in the interferometers cannot be neglected and, moreover, since they have approximately the same values as the even-numbered inductances $\left(L_{4-16}\right)$ along the transmission line, the high- $T_{c}$ de/SFQ converter exhibits a much more complicated dynamic behavior.

For the numerical simulations we have used a special program (written in "C" language) which can be compiled either for IBM compatible computers or (using the ANSI standard) for UNIX operated systems. The simple RSJ model without capacitance is employed because the experimental $I V$ curves did not show any hysteresis.

Figure 3(a) shows the simulated $I V$ curve of the parallel biased dc/SFQ converter and RSFQ transmission line with and without applied if power at a frequency of $70 \mathrm{GHz}$. Steps corresponding to the fundamental Josephson relation 

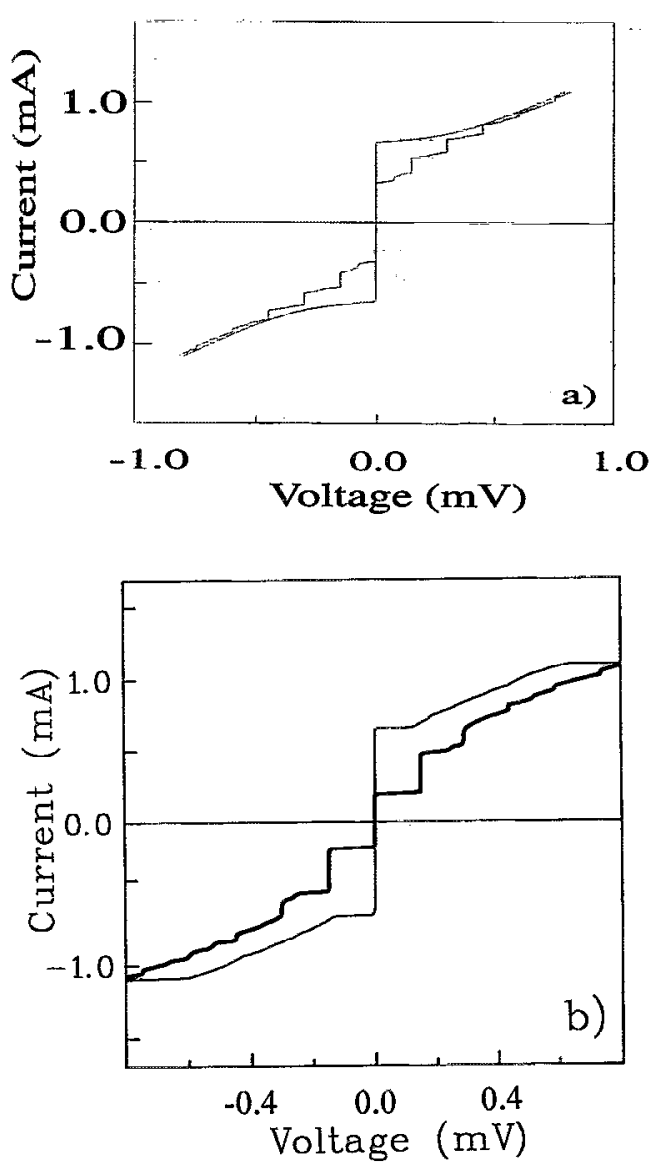

FIG. 3. IV curve of the de/SFQ converter with applied rf power (thick line) and without applied if power (thin line). (a) Simulation with an if current amplitude of $0.75 \mathrm{~mA}$. (b) Experimental $I V$ curve with $1 \mathrm{~mW}$ if power applied at the top of the sampleholder. $T=3.7 \mathrm{~K}$.

and their higher harmonics appear. The threshold level of the $\mathrm{dc} / \mathrm{SFQ}$ converter depends on the applied bias current, $I_{b}$. At least two operating regimes are available with the rf current $I_{\mathrm{rf}}=0.75 \mathrm{~mA}$ chosen for the simulation in Fig. 3(a). If the converter is dc current biased on the first step (say, at the voltage $V=145 \mu \mathrm{V}$ with $I_{b} \simeq 0.46 \mathrm{~mA}$ ), only one fluxon per if cycle is sent by the de/SFQ converter to the transmission line. At a higher bias current (e.q., around $I_{b} \simeq 0.63 \mathrm{~mA}$ ) the threshold level becomes smaller and two fluxons per rf period are launched onto the transmission line. This gives rise to the second step on the $I V$ curve at $V=290 \mu \mathrm{V}$.

The operational margins of the dc/SFQ converter as derived from the size (in bias current) of the steps as function of the applied $\mathrm{rf}$ power give an estimate for the stability of the device. Figure 4(a) shows the step size and position versus if current amplitude found by simulation. For the different operational regimes (i.e., one, two, or three fluxons per if cycle) it is then possible to find the optimum bias conditions in terms of $I_{b}$ and $I_{\mathrm{rf}}$. The critical current $I_{c 0}=0.65 \mathrm{~mA}$ sets an upper bias current limit for the operation with no applied rf signal.

The temporal operation of the converter is illustrated in Figs. 5(a)-5(c) and Figs. 5(d) and 5(e) for two different regimes with the parameters $I_{\mathrm{rf}}=0.75 \mathrm{~mA}, I_{b} \simeq 0.46 \mathrm{~mA}$, and

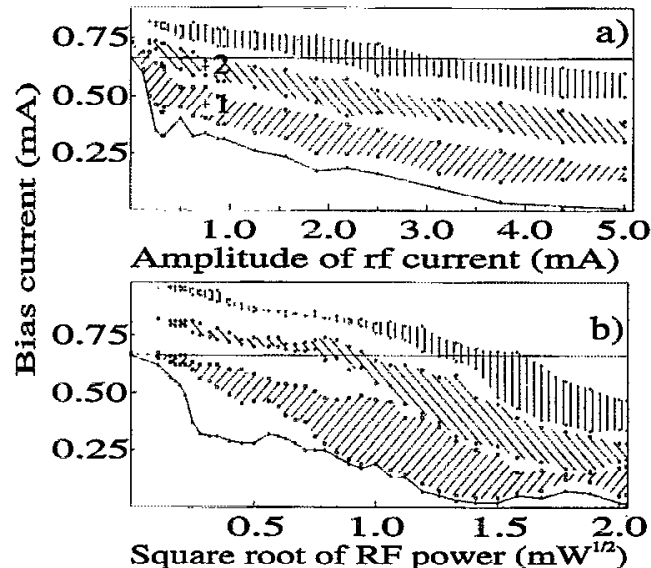

Square root of $R F$ power $\left(\mathrm{mW}^{1 / 2}\right)$

FIG. 4. Simulated (a) and experimental (b) dependence of the critical current and step positions (margins) for the first, the second, and the third step vs if signal. The two bias points marked +1 and +2 (for the rf current amplitude $I_{\mathrm{r}}=0.75 \mathrm{~mA}$ ) are used in the simulated results depicted in Figs. 5(a) $-5(\mathrm{c})$ and Figs. 5(d) and 5(f), respectively. The horizontal line marks the critical current $I_{c 0}=0.65 \mathrm{~mA}$ which without applied radiation is the upper limit for proper operation.

$I_{b} \approx 0.63 \mathrm{~mA}$, respectively, as indicated by the points " +1 " and " +2 " in Fig. 4(a).

Figure 5(a) shows the applied rf current for several cycles. The quantum mechanical phase in Fig. 5 is the difference between the superconducting phases at the electrodes of the Josephson junctions. Since all junctions are grounded at the same point, we can choose the phase at this electrode equal to zero. Then the quantum mechanical phase corresponds to the phase at the counterelectrode; for simplicity we shall use the term "phase." The phase is the time integral of the voltage across the junction according to the Josephson voltage-frequency relation. For the analysis of RSFQ circuits $^{1,8}$ it is practical to use the phase instead of the volt-

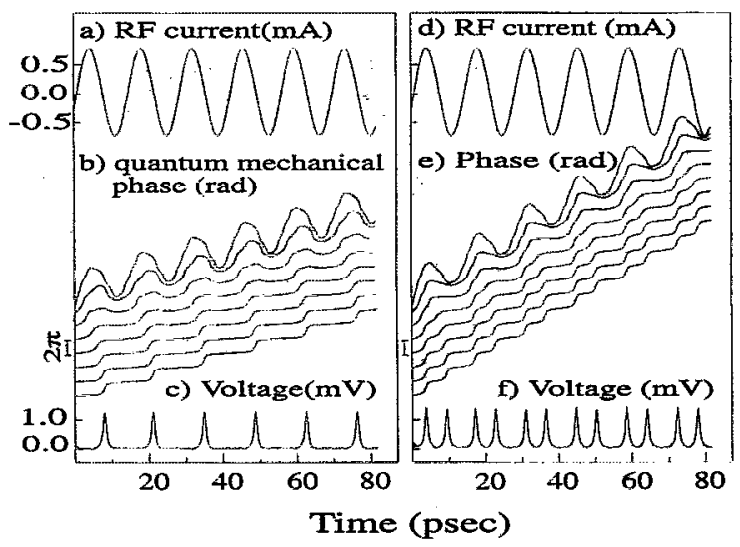

FIG. 5. Simulation of the de/SFQ converter for two different regimes. The amplitude of the rf current was $0.75 \mathrm{~mA}$ (a) and (d). The quantum mechanical phases (b) and (e) of junctions $J_{2}$ (top curve) to $J_{9}$ (bottom curve) are vertically shifted by $2 \pi$ for presentation. The voltage across $J_{9}$ (c) and (f) is the derivative of the phase curve for $J_{9}$. Cases (a) - (c) correspond to a bias current $I_{b}=0.46 \mathrm{~mA}$ and (d) $-(\mathrm{f})$ to $I_{b}=0.63 \mathrm{~mA}$, that were chosen to get symmetrical margins. 
age because often it is difficult to determine whether the voltage pulse across the junction actually switches the junction. On the phase diagram this can unambiguously be decided by the observation of a $2 \pi$ leap.

Figure $5(b)$ shows the phase at the junctions $J_{2}-J_{9}$ from top to bottom. As mentioned earlier the circuit in Fig. 2 displays a more complicated dynamic behavior than the $\mathrm{Nb}$ based circuit. During one rf cycle the phases at the junctions $J_{2}$ and $J_{3}$ exhibit a change of multiples of $2 \pi$, while the net change per cycle is $2 \pi$. This is due to the rather large inductances $L_{1-3}$ used to match the antenna to the converter. However the circuit $\mathrm{J}_{1-4}$ performs proper dc to SFQ conversion as seen from the phases at $J_{4}-J_{9}$. Figure 5(c) shows the voltage at junction $J_{9}$. The pulses correspond to one single flux quantum passing the junction per if cycle.

Figures $5(\mathrm{~d})-5(\mathrm{e})$ show the corresponding diagrams for the higher bias current $I_{b} \simeq 0.63 \mathrm{~mA}$. An interesting phenomenon appears in this two-fluxon regime. Even though the $\mathrm{dc} / \mathrm{SFQ}$ converter switches several times within the first halfcycle [see top curve in Fig. 5(e)], the time interval between the two fluxons released to the transmission line approaches a value equal to half of the if cycle during the propagation to junction $J_{9}$, as seen from the voltage pulses in Fig. 5(f). Thus it appears feasible to utilize this regime for the clocking of digital circuits at twice the operational frequency by using long transmission lines.

\section{EXPERIMENTAL RESULTS}

We have measured the $I V$ characteristic of the Josephson transmission line as function of applied rf power and temperature $T$. At $T=3.7 \mathrm{~K}$ with an estimated rf power at the antenna of about $0.25 \mu \mathrm{W}$ a small current step appears at a voltage corresponding to a fundamental Josephson oscillation at $70 \mathrm{GHz}$ for a bias current larger than the critical current of the unpumped junction, $I_{c 0}=0.65 \mathrm{~mA}$. In this case we observe the phase locking of the Josephson oscillations in the transmission line to the external radiation. The small value of the applied power corroborates the good coupling of the external generator to the Josephson circuit.

Figure 3(b) shows the $I V$ curve with and without applied microwave power. When the if current exceeds the threshold of the dc/SFQ converter, one observes a large step in the $I V$ curve at a voltage $V=145 \mu \mathrm{V}$ corresponding to $70 \mathrm{GHz}$ using the Josephson frequency relation. The critical current of the pumped junction is suppressed significantly below the critical current $I_{c 0}$. The size of the step is about $40 \%$ of $I_{c 0}$ at a power level of about $0.2 \mathrm{~mW}$. This gives a margin for the bias current of more than $\pm 20 \%$. An increased power produces higher harmonic constant-voltage steps corresponding to the launching of two or even three SFQ pulses per if cycle. The results are summarized in Fig. 4(a) which also gives the operational regime of the $\mathrm{dc} / \mathrm{SFQ}$ converter as a digital device.

With a power level chosen to optimize the size of the first step at low temperature the device operates to $T=66 \mathrm{~K}$. A typical $I V$ curve obtained at this temperature is shown in Fig. 6. At higher temperatures the critical current becomes too small to provide the necessary relation between the currents and the inductances for maintaining the operation of the

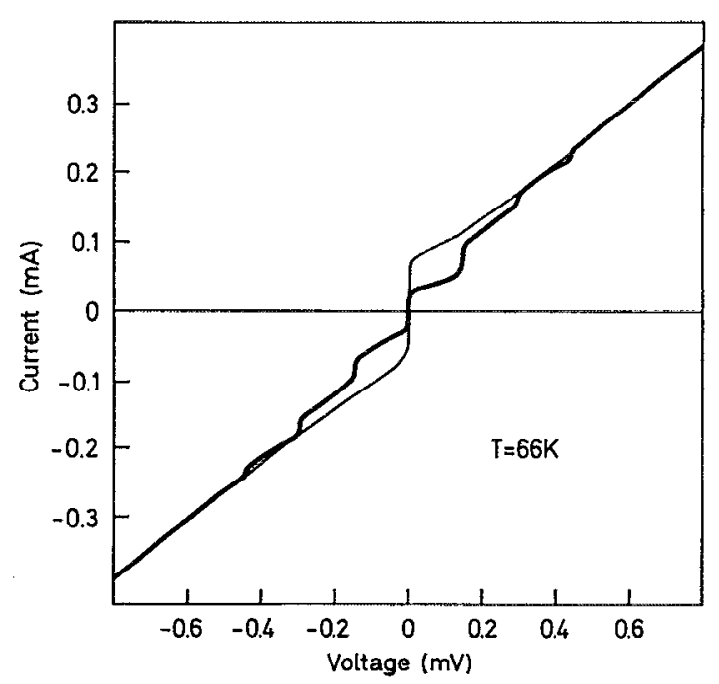

HIG. 6. Experimental de $I V$ curve for the dc/SFQ converter at $T=66 \mathrm{~K}$ without (thin line) and with applied (thick line) if power.

RSFQ circuit. No significant change in the $I V$ curve was observed around the superconducting transition temperature of the lead ground plane.

For an applied power as large as $4 \mathrm{~mW}$ we observe steps up to a voltage range corresponding to $1.5 \mathrm{THz}$ with bias currents as large as $3 \mathrm{~mA}$ at $T=4 \mathrm{~K}$. After the bias current reaches this high value the shape of the $I V$ curve changes, and the step height alters in a nonreproducible manner. The large bias current seems to induce magnetic fields into the superconducting electrodes.

\section{CONCLUSIONS}

We have designed, simulated, and experimentally tested a high- $T_{c}$ superconductor based dc to a single flux quantum converter that is loaded by a Josephson transmission line and coupled via a slot antenna to a millimeter wave $(\approx 70 \mathrm{GHz})$ external generator. The agreement between experiment and numerical simulation confirms the digital operation of the device. Testing of the dc/SFQ converter for RSFQ circuits at $70 \mathrm{GHz}$ is in progress using a Josephson junction based sampler, ${ }^{14}$ which directly can measure the stability of the repetition rate of the SFQ pulses at the output of the dc/SFQ converter.

\section{ACKNOWLEDGMENTS}

We are grateful to Dr. M. A. Tarasov for helpful discussions. This work was supported in parts by NORPAS, the Swedish-Russian Academy of Sciences cooperation, the Danish Research Academy, ESPRIT (Project No. 7100), NATO (LG 921040), and the EU program "Human Capital and Mobility."

\footnotetext{
${ }^{1}$ K. K. Likharev and V. K. Semenov, IEEE Trans. Appl. Supercond. 1, 13 (1991).

${ }^{2}$ V. K. Kaplunenko, M. I. Khabipov, V. P. Koshelets, K. K. Likharev, O. A. Mukhanov, I. L. Serpuchenko, and A. N. Vystavkin, IEEE Trans. Magn. MAG-25, 861 (1989).

${ }^{3}$ V. K. Kaplunenko, L. V. Filipenko, M. I. Khabipov, V. P. Koshelets, K. K.
} 
Likharev, S. V. Rylov, V. K. Semenov, and A. N. Vystavkin, IEEE Trans. Magn. MAG-27, 2464 (1991).

${ }^{4}$ O. A. Mukhanov, IEEE Trans. Appl. Supercond. 3, 2578 (1993).

${ }^{5}$ S. P. Benz, C. J. Burroughs, and C. A. Hamilton, IEEE Trans. Appl. Supercond. 3, 2582 (1993).

${ }^{6} \mathrm{Y}$. K. Kwong and V. Nandakumar, IEEE Trans. Appl. Supercond. 3, 2666 (1993).

${ }^{7}$ J. Fleischman and T. Van Duzer, IEEE Trans. Appl. Supercond. 3, 2716 (1993).

${ }^{8}$ S. V. Polonsky, V. K. Semenov, P. I. Bunyk, A. F. Kirichenko, A. Yu. Kidiyarova-Shevchenko, O. A. Mukhanov, P. N. Schevchenko, D. Yu. Zinoviev, and K. K. Likharev, IEEE Trans. Appl. Supercond. 3, 2566 (1993).

${ }^{9}$ J. S. Martens, J. R. Wendt, V. M. Hietala, D. S. Ginley, C. I. H. Ashby, T. A. Plut, G. A. Vawter, C. P. Tigges, M. P. Siegal, S. Y. Hou, Julia M. Phillips, and G. K. G. Hohenwarter, J. Appl. Phys. 72, 5970 (1992).

${ }^{10}$ T. Claeson, Z. G. Ivanov, V. K. Kaplunenko, E. A. Stepantsov, A. Ya.
Tzalenchuk, and E. Wikborg. Extended Abstracts of ISEC'93, Boulder, CO, 1993, pp. 78-79.

${ }^{11}$ Y. M. Zhang, D. Winkler, and T. Claeson, Appl. Phys. Lett. 62, 3195 (1993).

${ }^{12}$ B. Bi, S. Han, J. E. Lukens, and K. Wan, IEEE Trans. Appl. Supercond. 3, 2303 (1993).

${ }^{13}$ J. S. Martens, A. Pance, K. Char, S. Whiteley, and V. M. Hietala, Appl. Phys. Lett. 63, 1681 (1993).

${ }^{14}$ V. K. Kaplunenko, M. I. Khabipov, and E. B. Goldobin, Supercond. Sci. Technol. 4, 674 (1991).

${ }^{15}$ E. B. Goldobin, V. M. Golomidov, V. K. Kaplunenko, M. I. Khabipov, D. Yu. Khokhlov, and A. Yu. Kidiyarova-Shevchenko, IEEE Trans. Appl. Supercond. 3, 2641 (1993).

${ }^{16}$ Z. G. Ivanov, P. A. Nilsson, D. Winkler, J. A. Nlarco, T. Claeson, E. A. Stepantsov, and A. Ya. Tzalenchuk, Appl. Phys. Lett. 59, 3030 (1991). 